\title{
Sobrevida de Pacientes Pediátricos con Cardiopatía Congénita operados en Hospital María, Especialidades Pediátricas en 2017.
} Survival of Pediatric Patients with Congenital Heart Disease Operated at Hospital María, Especialidades Pediátricas in 2017.

David R. Díaz Rodríguez*(D), Josué A. Vásquez Alvarado*(D), Sonia Solórzano Gutiérrez**iD, Dilcia Sauceda Acosta***(D), Dr. Víctor Paz Flores ${ }^{* * * *}$, Dra. Dina Álvarez Corrales ${ }^{* * *}$ (D)

${ }^{*} \mathrm{MD}$, Especialista en Pediatría, Universidad Nacional Autónoma de Honduras

**MD, Jefe de Servicio de Cardiología, Hospital María, Especialidades Pediátricas

${ }^{* * *} \mathrm{MD}, \mathrm{MSc}$, Docencia e Investigación, Hospital María, Especialidades Pediátricas

****MD, Cirugía Cardiovascular, Malformaciones Congénitas y Adquiridas, Jefe Servicio Cardiocirugía Hospital María, Especialidades Pediátricas

\section{RESUMEN}

Antecedentes: las cardiopatías congénitas son la principal causa de defectos anatómicos en los recién nacidos a nivel mundial. En las últimas décadas ha mejorado la sobrevida de los pacientes que requieren cirugías correctivas 0 paliativas, aún las de mayor complejidad. Objetivo: determinar la sobrevida de pacientes con cardiopatías congénitas operados en el Hospital María, Especialidades Pediátricas (HMEP) en el 2017.

Metodología: se trata de un estudio transversal con componente analítico de 120 pacientes intervenidos quirúrgicamente en el año 2017. Se estimó la tasa de sobrevida a 1 año posterior a la cirugía. Los datos fueron extraídos de los expedientes clínicos y fueron registrados en una base de datos de Microsoft Access, para el análisis de datos se utilizó STATA 15.0. Resultados: la tasa de sobrevida a 1 año fue del 87.5 por cada 100 cardiocirugías realizadas. Se encontró diferencias significativas entre los vivos y fallecidos para nivel socioeconómico, clasificación RACHS-1, y Escala ROSS. Además, se encontró asociación con mortalidad en aquellos pacientes que presentaron cardiopatías cianóticas, otras malformaciones congénitas y complicaciones durante la estancia hospitalaria.

Conclusiones: la tasa de sobrevida encontrada en este estudio está acorde con la literatura internacional. Se recomienda profundizar en nuevos estudios que establezcan factores de riesgo de mortalidad en estos pacientes.

\footnotetext{
Correspondencia:

Dra. Dina Alvarez

Docencia e Investigación, Hospital María, Especialidades Pediátricas,

Tegucigalpa, Honduras

dalvarez@hospitalmaria.org / +50422713395

Recibido 28 de Junio 2021, Aceptado 10 Noviembre 2021.
}

Palabras Clave: Cardiopatía Congénita, Cirugía Cardiovascular, Sobrevida.

\section{SUMMARY}

Background: congenital heart disease is the leading cause of anatomical defects in newborns worldwide. In the recents decades the survival of patients who require corrective or palliative surgeries, even those of greater complexity, has improved.

Objective: to determine the survival of patients with congenital heart disease operated at the Hospital Maria, Especialidades Pediátricas (HMEP) in 2017.

Methods: this is an analytical cross-sectional study of 120 patients surgically operated in 2017. The survival rate was estimated at one year after surgery. The data was extracted from the clinical records and were registered in a Microsoft Acces database, for data analysis STATA 15.0 was used.

Results: the 1-year survival rate was 87.5 per 100 heart surgeries performed. Significant differences were found for socioeconomic level, RACHS-1 classification, and ROSS scale. In addition, an association with mortality was found in those patients who presented cyanotic heart disease, congenital malformations and complications during hospital stay. Conclusions: the survival rate found in this study is accordance with the international literature. It is recommended to deepen in new studies that establish mortality risk factors in these patients.

Keywords: Congenital heart disease, Cardiovascular Surgery, Survival. 


\section{Introducción}

Las cardiopatías congénitas (CC) corresponden a aquellas entidades que por definición son anomalías estructurales del corazón y/o grandes vasos intratorácicos y que están presentes desde el nacimiento, producidos por defectos en el mismo durante el periodo embrionario. En diferentes estudios se evidencia un aumento en la prevalencia de $\mathrm{CC}$ a nivel mundial, pasando de 0.6 por 1000 nacidos vivos en 1930 a 9.4 por 1000 nacidos vivos en el período de 2010 a 2017 esto en parte se debe a la mejoría de técnicas diagnósticas $(1,2$.).

En Latinoamérica, de acuerdo con el reporte del Estudio Colaborativo Latinoamericano de Malformaciones Congénitas (ECLAMC) la frecuencia de cardiopatías congénitas es de 2.6 por 10,000 aunque los datos en la región son variables de un país a otro (3). Además, se estima que sin ningún tipo de intervención médica el $14 \%$ de los pacientes no sobrevive al primer mes de vida y el $30 \%$ al primer año. La tendencia actual es la realización de cirugía precoz y correctora, o paliativa realizadas antes del 1 año de vida para mejorar la morbimortalidad $(4,5)$. Honduras por su parte cuenta con publicaciones que indican una incidencia de CC de 2.66 por cada 1000 nacidos vivos en el año 2006 en pacientes atendidos en el Instituto Hondureño de Seguridad Social (6).

En nuestro medio, los pacientes con estas patologías tienen oportunidades de diagnóstico y tratamiento limitadas debido a la escasez de personal especializado y tratamientos quirúrgicos disponibles. A partir del año 2016 el HMEP inicia el programa nacional de Cardiocirugía y ya para el 2020 se han realizado en total 941 procedimientos (7). En cuanto a la sobrevida de los pacientes postquirúrgicos está determinada por diversos factores como: tipo de cardiopatía, tiempo de diagnóstico, tipo de cirugía, lesiones residuales, presencia de cromosomopatías, estado nutricional, entre otros, Además existen diferentes métodos para estimar el riesgo de mortalidad según el tipo de cirugía, en este estudio utilizamos el método de estratificación de riesgo RACHS-1 (por sus siglas en inglés (Risk Adjustment in Congenital Heart Surgery) dividida en seis niveles, siendo el RACHS-1 el menor riesgo y el RACHS-6 el mayor (8). La información de la evolución de pacientes pos operados de Cardiocirugía publicada en nuestro medio es escasa, por lo que este estudio plantea un análisis de la sobrevida de pacientes pos operados de cardiopatías congénitas en Hospital María, Especialidades Pediátricas durante el año 2017.

\section{Metodología}

Realizamos un estudio transversal analítico en pacientes sometidos a cirugía cardiaca en HMEP que es un hospital público de referencia nacional para pacientes menores a 18 años, hasta el momento es la Institución que presta mayor atención clínico-quirúrgica a pacientes con cardiopatías congénitas. Durante el 2017, se realizó cirugía a 184 pacientes; para este estudio se excluyeron los pacientes a los cuales no se logró obtener acceso a su expediente clínico, mayores de 18 años al año después de haberse realizado la cirugía. Finalmente se tomaron en cuenta 120 pacientes para el análisis. (Figura No. 1)

\section{Número de Procedimientos realizados por Servicio de Cirugía Cardiovascular en $2017 \mathrm{~N}=198$}

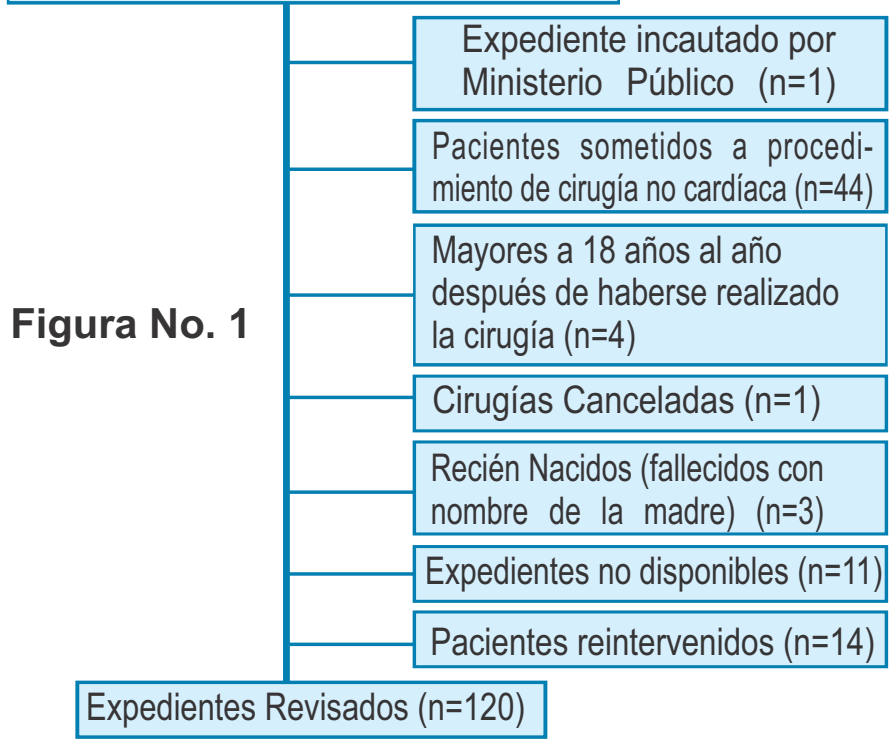

Previo al inicio de la investigación contamos con la aprobación del Comité de Bioética de Investigación del HMEP así como de las autoridades del mismo. Se realizó la revisión de los expedientes clínicos por los investigadores, registrando la información en la base de datos de Microsoft Access. El análisis de datos se realizó en STATA 15.0. Se calcularon medidas de frecuencia, medidas de tendencia central y 
dispersión. Para variables cualitativas se utilizó prueba de chi cuadrado con el objetivo relación de variables entre grupos de pacientes (vivos y fallecidos), para variables cuantitativas se utilizó prueba t de Student y diferencia de proporciones, además se calcularon Odds ratios de prevalencia para establecer asociación entre variables. Se estimaron intervalos de confianza (IC) al 95\%.

\section{Resultados}

Se estudiaron un total de 120 casos, de los cuales 15 fallecieron dentro del primer año posterior a la cirugía. La tasa de sobrevida fue del 87.5 por cada 100 cirugías. Dentro de las características epidemiológicas encontramos que los varones son ligeramente más afectados $(50.4 \%)$ que las niñas, el $47.6 \%$ son pre-escolares, el $42.5 \%$ de los pacientes proceden de Francisco Morazán, seguido de Cortés y Olancho con el $11 \%$, los departamentos con menor procedencia fueron Atlántida, Gracias a Dios, Yoro, Copán, Santa Bárbara, La Paz, Ocotepeque y Valle con porcentajes entre el $1 \%$ y $4 \%$. Se destaca que se encontraron diferencias estadísticamente significativas para los pacientes con nivel socioeconómico bajo y su condición, lo que significa que hay relación entre estas variables. El grupo con nivel socioeconómico bajo fue el que presentó mayor mortalidad. El cuadro No. 1 resume los factores sociodemográficos.

\section{Cuadro No 1. Factores sociodemográficos de pacientes con cardiopatía congénita operados en Hospital María, Especialidades Pediátricas año 2017.}

\begin{tabular}{cccc}
\hline \multirow{2}{*}{$\begin{array}{c}\text { Variable } \\
\mathbf{n}=120\end{array}$} & $\begin{array}{c}\text { Vivos } \\
(\mathbf{n}=105)\end{array}$ & $\begin{array}{c}\text { Fallecidos } \\
(\mathbf{n}=15)\end{array}$ & $\mathbf{p}$ \\
\hline $\begin{array}{c}\text { Sexo } \\
\text { Hombre }\end{array}$ & $53(50.4)$ & $5(33.3)$ & 0.21 \\
\hline Mujer & $52(49.6)$ & $10(66.7)$ & \\
\hline Edad Pediátrica & & & \\
\hline Neonato & $4(3.8)$ & $2(13.3)$ & \\
\hline Lactante menor & $27(25.7)$ & $7(46.7)$ & \\
\hline Lactante mayor & $11(10.4)$ & $1(6.7)$ & \\
\hline Preescolar & $36(34.3)$ & $2(13.3)$ & \\
\hline Escolar & $16(15.2)$ & $2(13.3)$ & \\
\hline Adolescente & $11(10.5)$ & $1(6.7)$ & \\
\hline Nivel Socioeconómico & & \\
\hline No Pobre & $23(21.9)$ & $0.03^{*}$ \\
\hline Pobre & $65(61.9)$ & $15(100)$ & \\
\hline $\begin{array}{c}\text { Bajo la Línea } \\
\text { de la Pobreza }\end{array}$ & $13(12.4)$ & $0(0)$ & \\
\hline Pobreza Extrema & $4(3.8)$ & $0(0)$ & \\
\hline${ }^{*}$ Estadísticamente significativo $p<0.05$, Fuente: encuesta. \\
\hline
\end{tabular}

La edad al diagnóstico tuvo una media de 25 meses para los pacientes vivos y 24 para los fallecidos, dentro de este grupo el $67 \%$ correspondieron a Cardiopatías cianóticas, encontrando que el $60 \%$ estaba asociado a otras malformaciones extracardíacas. El tiempo promedio para la realización de la cirugía de los pacientes que sobrevivieron fue de 33 meses posterior al diagnóstico. El tipo de cardiopatía congénita que más se intervino fueron las cardiopatías congénitas acianóticas en un $75 \%$ como Coartación de Aorta, PCA y CIV. EI 34\% de las cirugías fueron en pacientes con cardiopatías cianóticas, siendo el diagnóstico más frecuente la Tetralogía de Fallot; se encontraron diferencias significativas entre ambos grupos.

Para la clasificación de riesgo quirúrgico se utilizó la escala RACHS-1 siendo 1 el riesgo más bajo y 6 el más alto. Encontramos que el mayor número de pacientes se catalogó dentro de la escala RACHS-2 (35\%) con una sobrevida del $92.8 \%$. RACHS 5 y 6 no fueron intervenidos en el 2017. La genopatía encontrando con más frecuencia fue el Síndrome de Down (12.5\%) de los cuales el $40 \%$ falleció. De acuerdo con la clasificación ROSS, que estratifica el grado de severidad de insuficiencia cardiaca el $71.6 \%$ corresponde al grado I. En cuanto a la estancia hospitalaria en días se encontró una media de 12 días para los pacientes vivo y 13 días fallecidos, no encontrando diferencias significativas entre ambos grupos. Se encontró diferencias para tiempo en ventilador mecánico y tiempo de camplaje aorta (TCA), otras variables relacionadas con la estancia hospitalaria se encuentran descritas en cuadro No. 2. 
Cuadro No. 2. Características de atención en pacientes operados de cardiopatía congénita en HMEP año 2017.

\begin{tabular}{|c|c|c|c|}
\hline \multirow[t]{2}{*}{ Variable } & \multicolumn{2}{|c|}{ Condición } & \multirow[b]{2}{*}{ p } \\
\hline & Vivos & Fallecidos & \\
\hline $\begin{array}{l}\text { Edad al diagnóstico } \\
\text { (meses) }\end{array}$ & 25 & 24 & $0.00^{*}$ \\
\hline $\begin{array}{c}\text { Edad a la cirugía } \\
\text { (meses) }\end{array}$ & 33 & 16 & 0.89 \\
\hline $\begin{array}{c}\text { Estancia } \\
\text { intrahospitalaria } \\
\text { (días) }\end{array}$ & 12 & 13 & 0.66 \\
\hline $\begin{array}{l}\text { Estancia en UCI } \\
\text { (días) }\end{array}$ & 4 & 7 & 0.21 \\
\hline $\begin{array}{l}\text { Estancia en sala } \\
\text { (días) }\end{array}$ & 7 & 6 & 0.48 \\
\hline $\begin{array}{l}\text { Tiempo de Ventilador } \\
\text { mecánica (días) }\end{array}$ & 2 & 7 & $0.02^{*}$ \\
\hline $\begin{array}{l}\text { Tiempo ECMO** } \\
\text { (minutos) }\end{array}$ & 46 & 129 & 0.15 \\
\hline $\begin{array}{l}\text { Tiempo de TCA } \\
\text { (minutos) }\end{array}$ & 28 & 69 & $0.00^{*}$ \\
\hline \multicolumn{4}{|l|}{$\begin{array}{l}\text { Tipo de Cardiopatía } \\
\text { Congénita }\end{array}$} \\
\hline $\begin{array}{l}\text { Cianótica de Flujo } \\
\text { Pulmonar Aumentado }\end{array}$ & $6(5.7)$ & $4(26.7)$ & $0.01^{*}$ \\
\hline $\begin{array}{l}\text { Cianótica de Flujo } \\
\text { Pulmonar Disminuido }\end{array}$ & $25(23.8)$ & $6(40)$ & \\
\hline $\begin{array}{l}\text { Acianótica de Flujo } \\
\text { Pulmonar Aumentado }\end{array}$ & $70(66.7)$ & $5(33.3)$ & \\
\hline $\begin{array}{l}\text { Acianótica de Flujo } \\
\text { Pulmonar Normal }\end{array}$ & $4(3.8)$ & $0(0)$ & \\
\hline \multicolumn{4}{|l|}{ Tipo de Cirugía } \\
\hline Correctiva & $95(90.4)$ & $13(86.7)$ & 0.65 \\
\hline Paliativa & $10(9.6)$ & $2(13.3)$ & \\
\hline \multicolumn{4}{|l|}{ Riesgo RASCH } \\
\hline 1 & $43(41)$ & $2(13.3)$ & $0.00^{*}$ \\
\hline 2 & $39(37.1)$ & $3(20)$ & \\
\hline 3 & $21(20)$ & $7(46.7)$ & \\
\hline 4 & $2(1.9)$ & $3(20)$ & \\
\hline \multicolumn{2}{|l|}{$\begin{array}{l}\text { Malformación } \\
\text { Congénita Asociada }\end{array}$} & & $0.00^{*}$ \\
\hline Si & $12(11.4)$ & $9(60)$ & \\
\hline No & $93(88.6)$ & $6(40)$ & \\
\hline \multicolumn{4}{|c|}{ Clasificación de ROSS } \\
\hline 1 & $85(81)$ & $1(6.7)$ & $0.00^{*}$ \\
\hline II & $16(15.2)$ & $1(6.7)$ & \\
\hline III & $2(1.9)$ & $8(53.3)$ & \\
\hline IV & $2(1.9)$ & $5(33.3)$ & \\
\hline
\end{tabular}

* Estadísticamente significativo $p<0.05$,

** Oxigenación por membrana extracorpórea.

Fuente: encuesta
El $59 \%$ de los pacientes presentaron complicaciones posteriores a la intervención quirúrgica. Dentro de las complicaciones cardíacas el síndrome de bajo gasto postoperatorio se presentó en $41.3 \%$ de los pacientes y disfunción ventricular en el $13.7 \%$; de las complicaciones no cardíacas el derrame pleural se presentó en $23.5 \%$, neumotórax en $21.5 \%$, y choque hipovolémico en un $13.7 \%$. Como resultado de estas complicaciones el $10.8 \%$ presentó parada cardiorrespiratoria. El $46.6 \%$ de los pacientes que fallecieron lo hicieron en las primeras 24 horas y el $53.4 \%$ falleció antes de los 30 días posterior a su cirugía catalogándose como muertes tempranas. Este estudio encontró que las cardiopatías cianóticas tienen mayor probabilidad de mortalidad que los pacientes con cardiopatías acianóticas. Asimismo, los pacientes con malformaciones congénitas y complicaciones durante su estancia hospitalaria. Ver cuadro No. 3.

Cuadro No. 3. Factores asociados a defunción en pacientes operados de cardiopatía congénita en HMEP, año 2017

\begin{tabular}{|c|c|c|c|c|c|c|}
\hline \multirow{3}{*}{ Factores } & \multicolumn{4}{|c|}{ Condición } & \multirow{3}{*}{ OR $p \quad$ IC $95 \%$} & \multirow{3}{*}{$p$} \\
\hline & \multicolumn{2}{|c|}{ Fallecidos } & \multicolumn{2}{|c|}{ Vivos } & & \\
\hline & n & $\%$ & $\mathbf{n}$ & $\%$ & & \\
\hline \multicolumn{7}{|c|}{ Tipo de cardiopatía } \\
\hline Acianótica & 5 & 33 & 74 & 71 & \multirow{2}{*}{$0.21 \quad 0.05-0.75$} & \multirow{2}{*}{0.00} \\
\hline Cianótica & 10 & 67 & 31 & 29 & & \\
\hline \multicolumn{7}{|c|}{ Malformaciones } \\
\hline $\mathrm{Si}$ & 9 & 60 & 11 & 11 & \multirow{2}{*}{$12.813 .25-51.4$} & \multirow{2}{*}{0.00} \\
\hline No & 6 & 40 & 94 & 89 & & \\
\hline \multicolumn{7}{|c|}{ Complicaciones } \\
\hline $\mathrm{Si}$ & 14 & 93 & 45 & 43 & \multirow{2}{*}{$18.672 .61-801$} & \multirow{2}{*}{0.00} \\
\hline No & 1 & 7 & 60 & 57 & & \\
\hline
\end{tabular}

Fuente: Encuesta

\section{Discusión}

En el presente estudio la tasa de sobrevida en la población sometida a procedimiento quirúrgico por presentar malformación congénita cardíaca fue de $87.5 \%$ a un año. Esta tasa de sobrevida es mayor a la presentada en otros estudios como ser en el estudio de García et al. (9), realizado en México en donde la tasa de sobrevida a 1 año postquirúrgico fue de $44 \%$; siendo similar en otros estudios a este como lo publicado por 
Magliola et al. (10), quien reportó una tasa de sobrevida del $88 \%$ así como Wren et al. (11) quienes encontraron sobrevida de $82 \%$ y $93.7 \%$ descrito por Jenkins et al (14); se encontraron también otros estudios latinoamericanos como el publicado por Rodríguez et al (12) en donde encontraron sobrevida de $79.3 \%$ en neonatos con cardiopatía compleja.

Queda manifestada la gran importancia de realizar cirugías tempranamente, encontrando en este estudio que el tipo de cirugía correctiva tiene cifras más altas de sobrevida a un año en comparación con la paliativa, con $88 \%$ y $83 \%$ respectivamente. Es importante destacar que en esta investigación se encontraron cifras mayores a lo encontrado en otras investigaciones como la de Magliola et al.(10) en donde para las cirugías paliativas se apreció una sobrevida cercana al $58 \%$. Esto podría explicarse por las altas listas de espera para cirugía lo que provoca manejo tardío; ya que se diagnostican e intervienen a los pacientes hasta un año posterior al nacimiento. En este estudio se encontró que la intervención se realizó en alrededor de 16 meses posteriores al diagnóstico, cuando los tiempos quirúrgicos ya no son favorables y resulta muy difícil cambiar el desenlace fatal $(12,13,14)$.

Aunque el puntaje RACHS-1 es un buen predictor de mortalidad, no aborda los factores individuales y estructurales de un servicio que pueden afectar directamente los resultados quirúrgicos. En este estudio el $96.8 \%$ de los pacientes se concentraron en los grupos quirúrgicos de más bajo riesgo (categoría 1,2,3) al igual que en otros estudios como Clavería et al (15), en donde el $90 \%$ de sus procedimientos se encontraron en las mismas categorías por ende con tasas más altas de sobrevida. Los procedimientos de más alto riesgo (categorías 4,5 y 6 ) representan solamente $4.1 \%$ de los pacientes intervenidos pero con una mortalidad del $60 \%$, siendo esta alta al igual que en el estudio de Clavería et al en donde presentaron sólo el $12 \%$ del total de procedimientos y el $45 \%$ de toda las defunciones (15). En este estudio el riesgo quirúrgico fue determinado por la clasificación de RASCH-1 encontrando tasas de mortalidad de $4.5 \%$ en la categoría $1 ; 7.2 \%$ en categoría 2; $25 \%$ en categoría 3; y $60 \%$ en la categoría 4; no se intervinieron pacientes correspondientes a categoría 5 y 6 . En una investigación realizada en São Paulo (7) en 2011 la mortalidad fue de $0.9 \%$; $4.7 \%$; $8.5 \%$; $20.4 \% ; 42.9 \%$ y $50 \%$, en la categorías $1,2,3,4$, 5 y 6 , respectivamente, mostrando una disminución en sus porcentajes de mortalidad, desestimando otras afecciones presentadas por los pacientes al momento de la intervención (16).

En un estudio realizado en Bélgica en paciente operados por cirugías complejas como las cirugías realizadas para la corrección de transposición de grandes arterias después de 30 años de haber realizado procedimientos. Se encontró que de 339 pacientes estudiados, $24.2 \%$ murieron durante el posoperatorio inmediato o tardío; la mortalidad temprana se dio en el $16.5 \%$ de los pacientes y mortalidad tardía se dio en el $7.7 \%$ de los casos (17). En nuestro estudio no se ahondó sobre factores relacionados con mortalidad temprana o tardía y deben ser considerados para estudios posteriores. En los países en desarrollo, además de la complejidad del procedimiento, deben tenerse en cuenta otros determinantes al evaluar la mortalidad. Aproximadamente el 90\% de los nacimientos en todo el mundo ocurren en estos países y solo una pequeña parte de la población $(7 \%)$ tiene acceso al tratamiento de cardiopatías congénitas, debido a la falta de recursos en el área, lo que lleva a un aumento de la morbilidad y la mortalidad $(18,19)$ como en nuestro estudio en donde se encontró retraso en las intervenciones donde el promedio los pacientes tendrían que esperar hasta 32.9 meses antes de lograr ser intervenidos. Otro factor en la población que falleció fue el tiempo de estancia en TCA encontrando tiempos promedios de 129.3 minutos y tiempos de circulación extracorpórea y 68.7 minutos siendo establecido de forma general que un tiempo de pinzamiento aórtico inferior a los 60 minutos y un tiempo circulación extracorpórea (TCEC) menor de 90 minutos presenta una menor mortalidad en los casos. Este estudio no hizo énfasis en factores de mortalidad quirúrgica temprana y tardía que podrían dar mejores resultados para la comprensión de los pacientes fallecidos $(20,21)$. La limitante principal de este estudio fue la utilización de fuente secundaria como fuente 
de datos, que por consiguiente los datos faltantes no pueden ser recuperados. De igual forma la falta de disponibilidad de algunos expedientes clínicos redujo la cantidad de datos obtenidos. De igual manera, la cantidad no muy robusta de pacientes estudiados limitó el análisis para variables muy importantes como ser: tasa de sobrevida por tipo de cardiopatía y tipo de corrección quirúrgica. A pesar de las limitantes consideramos que se aportan datos que pueden servir para la formulación de hipótesis en estudios futuros.

\section{Conclusión:}

Encontramos una tasa global de sobrevida del $87.5 \%$ en aquellos pacientes que fueron intervenidos quirúrgicamente, con pronóstico desfavorables aquellos cuya complejidad era superior según el calificativo que otorga la puntuación RASCH1, lo que es acorde a la literatura disponible. Los factores dependientes desfavorables que se involucran significativamente en la mortalidad de los pacientes operados se conducen en base a la variante anatómica de cardiopatías cianóticas de flujo pulmonar aumentado o disminuido, esto implica cirugías de mayor complejidad, así como el prolongado tiempo de espera entre el diagnóstico y la cirugía. Se hacen necesarios más estudios que profundicen en factores asociados a mortalidad quirúrgica temprana y tardía en pacientes con CC.

\section{Declaración}

Los autores hemos asumido la responsabilidad del contenido y resultados. Los autores del manuscrito declaramos no tener conflicto de intereses.

\section{Agradecimientos}

ADios por sus misericordias cada día, al Hospital María, Especialidades Pediátricas por abrirnos las puertas.

\section{Conflicto de interés: Ninguno declarado}

\section{Bibliografía}

1. Linde DV, Konings EE; Slager M; Witsenburg $\mathrm{M}$; et all. Birth prevalence of congenital heart disease worldwide: a systematic review and meta-analysis. Journal of the American College of Cardiology 58(21), 2241 2247,2011.

2. Liu Y, Chen S, ZuhlkeL; Black GC, Choy;L. Li $\mathrm{N}$, Keavney BD. Global birth prevalence of congenital heart defects 1970-2017:update systematic review and meta-analysis of 260 studies. Journal Epidemiology. 2019 Apr $1 ; 48(2) ; 455-463$.

3. Nazer HJ, Cifuentes OL. Congenital malformations in Latin America in the period 1995-2008. Rev Medi Chil. 2011; 139-72-8. Disponible en :

4. Tassinari S; Martínez V.S; Erazo N; Pinzón MC; García G: Zarante I. Epidemiología de las cardiopatías congénitas en Bogotá Colombia, entre 2001-2014: ¿mejoría en la vigilancia o aumento en la prevalencia?. Biomédica 2018; 38-141-8.

5. Ruiz A; Castillo M; García S, Peiro C y col. Perspectiva actual de la cirugía de las cardiopatías congénitas. Rev. Española de Pediatría Vol 71 N5, 2015.

6. Carvajal-Sierra P, Aronne-Gulllen E. Incidencia de cardiopatías congénitas en recién nacidos en el Hospital Materno Infantil diagnosticados entre octubre 2001 y mayo 2003. Honduras Pediátrica. 2006; Vol XXVI(No. 2):12-16.

7. Hospital María, Especialidades Pediátricas. Tecnología Comunicación e Información. Departamento de Estadística. Servicio Cardiología 2017.

8. Calderón-Colmenero J, Ramírez MS, Cervantes SJ. Métodos de estratificación de riesgo en la cirugía de cardiopatías congénitas. Arch Cardiol Mex. 2008;78(1):60-67

9. García H, Ramos J, Villegas S, Rodríguez L, Vera C. Sobrevida al egreso hospitalario de recién nacidos con cardiopatías congénitas sometidos a cirugías cardiacas o cateterismo intervencionista. Rev Inv Clin 2002; 54 (4): 311-9.

10.Magliola R, Althabe M, Moreno G, Lenz AM, Pilan ML, Landry L. Cardiac surgical repair in 
newborns: five years' experience in neonatal open surgery. Arch Argent Pediatr 2009; 107 : 417-22.

11.Wren C, O'Sullivan JJ. Survival with congenital heart disease and need for followup in adult life. Heart 2001; 85: 438- 43.

12.Rodríguez GA, Soler RM, Jiménez AI, Ortiz $E A$, Expósito ZM. La supervivencia en neonatos con afecciones complejas en Cirugía Pediátrica. Correo Científico Médico. 2018 Oct 8;23.

13. Ministério da Saúde. [Internet] Sistema de informações hospitalares do SUS (SIH/SUS). [Accessed on: 15/06/2015]. Available at: http:/w3.datasus.gov.br/ datasus/datasus.php.

14.Jenkins $\mathrm{KJ}$, Castañeda $\mathrm{AR}$, Cherian $\mathrm{KM}$, Couser CA, Dale EK, Gauvreau K, et al. Reducing mortality and infections after congenital heart surgery in the developing world. Pediatrics. 2014;134(5):e1422-30.

15. Clavería C, Cerda J, Becker P, Schiele C, Barreno B, Urcelay $G$ et al. Mortalidad operatoria y estratificación de riesgo en pacientes pediátricos operados de cardiopatía congénita: experiencia de 10 años. Revista chilena de cardiología. 2014;33(1):11-19

16.Mayer M, Gold J. Postoperative heart failure after stage 1 palliative surgery for single ventricle cardiac disease. Ped cardiology. 2019; 40 (5): 943-949

17. Moons P; Gewilling M; Sluysmans T; Verhaaren $\mathrm{H}$; Viart $\mathrm{P}$ et all. Long term outcome up to 30 years after the Mustard or Senning operation:a nationwide multicentre study in Belgium. Interventional Cardiology and Surgery. Heart 2004; 90:307-313.

18.Burkhardt B, Rücker G, Stiller B. Prophylactic milrinone for the prevention of low cardiac output syndrome and mortality in children undergoing surgery for congenital heart disease. Chrocrane library. 2015. Issue 3.

19.Kuraim et al. Predictors and outcomes of early postoperative veno-arterial extracorporeal membrane oxygenation following infant cardiac surgery Journal of Intensive Care. 2018; 56(6): 1-12.
20.Agarwal H, Hardison D, Saville B, Donahue $B$, Lamb F, Bichell D. Residual lesions in postoperative pediatric cardiac surgery patients receiving extracorporeal membrane oxygenation support. J Thoracic Cardiovasc Surg. 2014;147(1):434-441.

21. Thiagarajan $R$. et al. Extracorporeal life support organization registry international report 2016. ASAIO J. 2017; 63:60-67. 